\title{
Color-Based Iris Verification
}

\author{
Emine Krichen, Mohamed Chenafa, Sonia Garcia-Salicetti, and Bernadette Dorizzi \\ Institut National des Télécommunications, 9 Rue Charles Fourier 91160 Evry France \\ \{emine.krichen, sonia.salicetti, bernadette.dorizzi\}@int-evry.fr
}

\begin{abstract}
In this paper we propose a novel iris recognition method for iris images acquired under normal light illumination. We exploit the color information as we compare the distributions of common colors between a reference image and a test image using a modified Hausdorff distance. Tests have been made on the UBIRIS public database and on the IRIS_INT database acquired by our team. Comparisons with two iris reference systems in controlled scenario show a significant improvement when using color information instead of texture information. On uncontrolled scenarios, we propose a quality measure on colors in order to select good images from bad ones in the comparison process.
\end{abstract}

Keywords: Iris recognition; Hausdorff distance; quality measure, Gaussian Mixture Models.

\section{Introduction}

Iris acquisition is a challenging task. Indeed, a small and in most of the cases dark object has to be acquired from a relative long distance (from $35 \mathrm{~cm}$ to $3 \mathrm{~m}$ ). Iris is also a moving target, hidden in another moving object (the eye) almost covered by eyelids, bounded nearly in the middle by a dark hole (the pupil) which can dilate and contract depending on the illumination intensity during the acquisition process. These dilations and contractions of the pupil induce changes in iris texture which unfortunately are nonlinear.

Nevertheless, the most challenging problem is that iris is located behind the cornea, a very high reflective mirror, which makes impossible to acquire irises in normal light without some constraints and extra tools. Figure 1 (left) shows an iris acquired under normal illumination conditions and without any particular constraint. Reflections do not permit any accurate processing in this case. One solution would be the use of a strong source of illumination on the eye (typically a flash). Figure 1 (middle image) shows the kind of images obtained using this technique with normal light illumination. Reflections are then mostly deleted, except the ones resulting from the use of the flash itself. Despite the relative good quality of this image, in general, these alternatives are not sufficient for a performant texture analysis.

In practice, near infrared illumination is considered to be the most appropriate for iris acquisition. Indeed, it allows tackling the problem of cornea reflection. Moreover, near infrared waves penetrate the cornea and allow getting very good quality iris images with all the richness of the iris texture, even with dark irises. Figure 1 (right 
image) shows an iris image acquired under near infrared illumination. As can be seen the image quality is substantially higher than the one of the two irises previously presented. All commercial iris solutions are running today on images acquired under this kind of illumination [1].

However, there are applicative situations where we have at disposal a color camera with more or less high resolution. This is the case on PDA and smartphones. Our aim, in this article is to study whether it is possible to use these cameras and more especially the color information of the iris to produce a system sufficiently accurate to perform iris identity verification.

Very few works report results on this type of images. In [2], we proposed a small modification of the standard iris techniques by using wavelet packets instead of the classic Gabor wavelet. We ran preliminary experiments on a private database using a 3 million pixels camera, indoor and with a flash (figure 1 (middle) is an image from such database). We also studied the contribution of color information to the wavelet method. We showed that the mean of the inter-class distribution using color information is higher than the one obtained with grey level images. In other publications [3] presenting experiments on normal light images, researchers just transform color irises into grey level ones and apply on this type of images the algorithms they developed previously for near infrared images.

Our paper is organised as follows: section 2 describes all the modules composing our proposed algorithm, including iris segmentation, normalization and recognition. In section 3 we introduce the databases on which we have run our experiments namely IRIS_INT and UBIRIS. UBIRIS [4] is an iris database acquired under normal light illumination. It is a public database, which contains about 250 persons, some of them with 2 sessions. In the second session, irises are acquired under uncontrolled mode (outdoor, uncontrolled illumination, ...). Experimental results will be given in section 4. Also, as benchmarking tools for our system, we consider two reference iris systems, Masek [6] (used by NIST) and OSIRIS, a new reference system developed by our team in the framework of BioSecure. We demonstrate that the use of color information can highly outperform methods only based on texture information. We also propose a global quality measure on color images in order to select test images during the matching process. Finally, conclusions are given and future works is discussed.
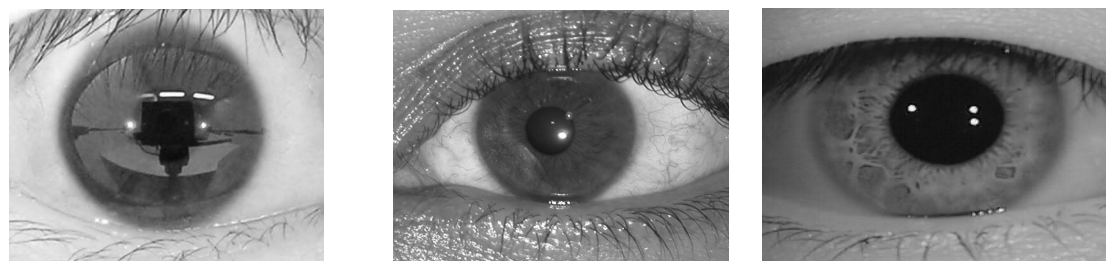

Fig. 1. Iris acquisition under normal light conditions without any restrictions (left image), normal light iris acquisition under a controlled scenario with a flash (middle image from IRIS_INT database) and near infrared iris acquisition (right image from CASIAv2 database) 


\section{Iris Recognition on Color Images}

The iris rim is segmented from original iris images using the Hough Transform method proposed and explained in [5] and developed in open source by Masek [6]. Once iris and pupil circles have been detected, we normalize the iris rim using the rubber sheet model proposed by J. Daugman in [7]. The segmentation and normalization processes are shown in Figure 2.
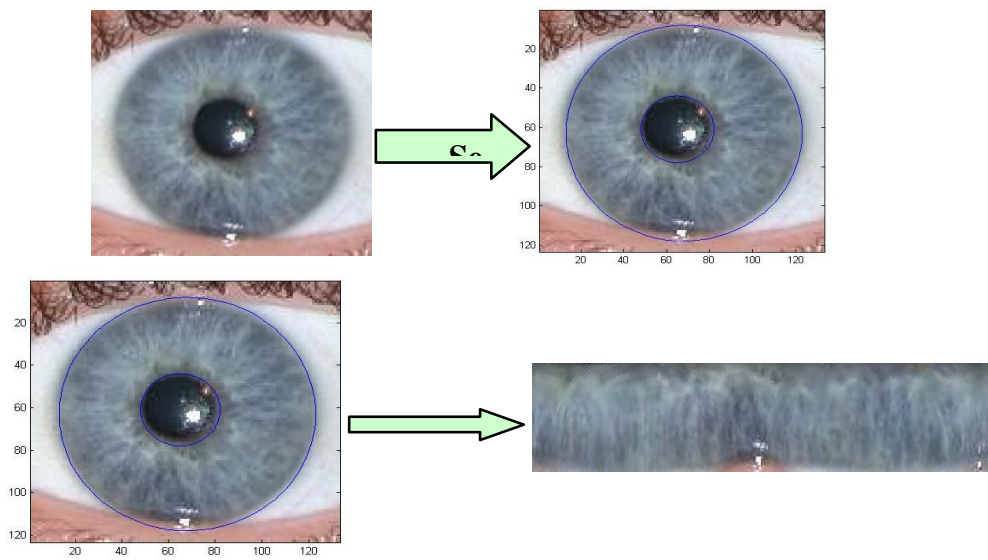

Fig. 2. Iris segmentation (Top images): the iris and pupil contours are considered as circles and are detected using the Hough Transform. Iris normalization (Bottom images): the iris is normalized using the rubber sheet model.

Our iris recognition method is composed of several steps: first we need to compress the colors present in the reference image into a fixed number of colors. We have used the minimum variance quantization in order to reduce the number of colors [9]. From each original image, we produce a compressed image with its corresponding color map (figure 3). Both information will be used in the next stages.

We need to compress the number of colors of the images because of the fact that at least 10.000 colors are present in the iris image even in dark and poorly textured irises. This makes any color-based method very heavy to develop.

Once the reference iris image is compressed, we process the test image using the color map obtained for the reference image. We assign each color in the map to the nearest color present in the test iris image using the 3 dimensional color cube (RGB space). Then, for each color in the map, we compare the distributions of the corresponding pixel positions in the reference and test images. This comparison is made using a modified Hausdorff Distance [8].

The Hausdorff Distance is an asymmetric distance which measures the maximum distance of each point of a set to the nearest point in the other set. Formula 1 shows the original expression of the Hausdorff Distance between set $X$ and set $Y$ where $x$ is an element of $X, y$ of $Y$ and $d$ denotes the Euclidian distance.

$$
d_{H}(X, Y)=\max \left\{\sup _{x \in X} \inf _{y \in Y} d(x, y), \sup _{y \in Y} \inf _{x \in X} d(x, y)\right\} .
$$




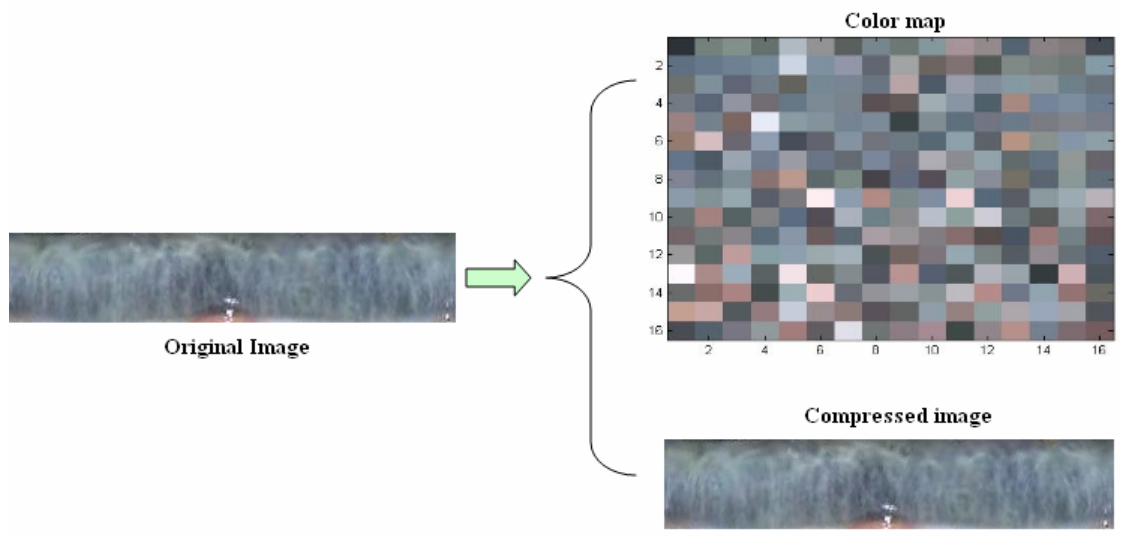

Fig. 3. Calculation of scores between Image 1 and Image 2 including: the compression of the two images; the creation of a set of pixels whose values are equal to each color and finally the computation of the Hausdorff Distance between set $\mathrm{X}$ and $\mathrm{Y}$

This formula of the Hausdorff distance has a great limitation if we want to apply it to our problem because it works at the pixel level; indeed, only one pixel that is an outlier can modify dramatically the result of the Hausdorff Distance. For instance, this will occur with pixels which correspond to noise as eyelids, spot light reflections, eyelashes ... for these reasons, we have used a modified form of the Hausdorff Distance in which we took the minimum between the Hausdorff distance calculated between $\mathrm{X}$ and $\mathrm{Y}$ on one hand and $\mathrm{Y}$ and $\mathrm{X}$ on another hand, and we computed the average between the Euclidian distances of elements of one set to the elements of the other set. This modified Hausdorff Distance is expressed in Formula 2:

$$
d_{H \text { mod }}(X, Y)=\min \left[\frac{1}{N x} \sum_{X} \inf _{x} d(x, y), \frac{1}{N y} \sum_{Y} \inf _{y} d(y, x)\right]
$$

Finally, the score between two images is computed by averaging the modified Hausdorff distances obtained for each color, on all possible colors c of the map (see Formula 3). Xc and Yc are the pixel positions corresponding to color c respectively in the reference image Iref and the test image Itest.

$$
S(\text { Iref }, \text { Itest })=\text { mean }_{c}\left(d_{H \text { mod }}\left(X_{c}, Y_{c}\right)\right)
$$

In order to accelerate our algorithm, only distributions with comparable pixels size are used for comparison. The whole recognition process is described in Figure 4.

Based on the dominant color of the iris, we made a coarse pre-classification between irises. We notice that two main classes of colors are present in the iris images (blue and brown images). The Hue distribution (form the HSV color space instead of RGB color space [10]) can be used to automatically classify the irises into 2 classes. The Hue value indicates the nature of the color and is expressed in terms of angle (degrees). For brown irises, the Hue distribution falls into the range of $0^{\circ}(90 \%$ of the 


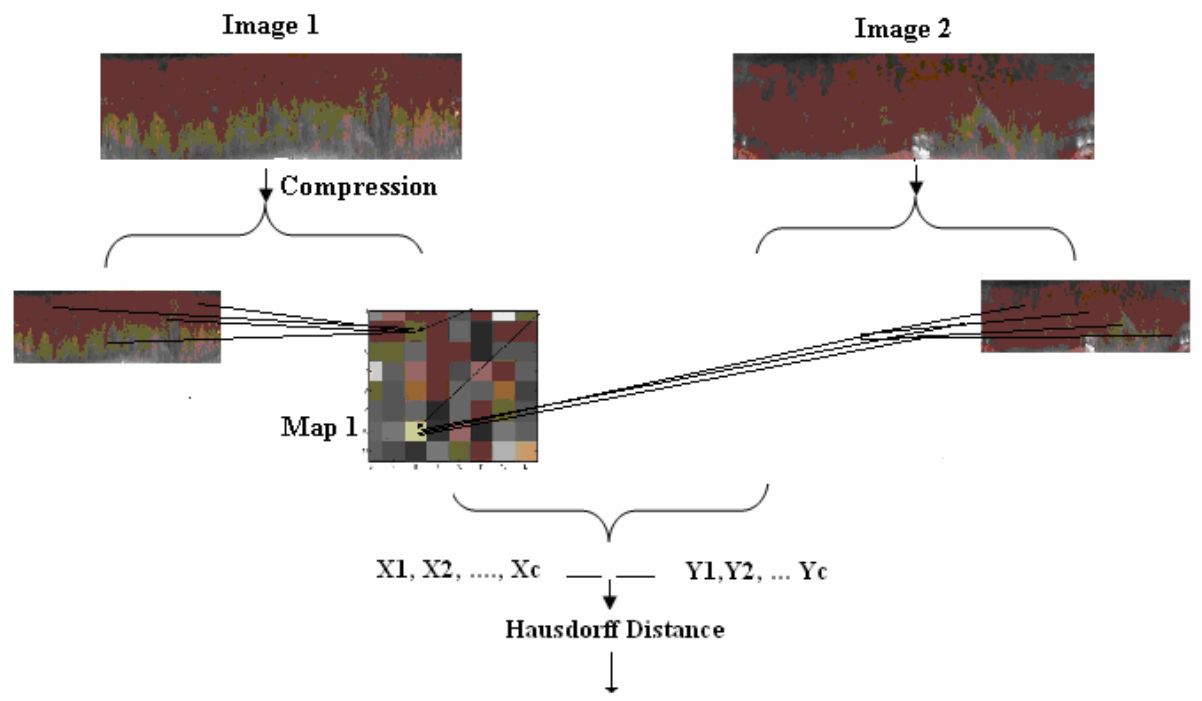

Final Score

Fig. 4. Scores computation between Image 1 and Image 2 including: the compression of the two images; the creation of a set of pixels whose values correspond to a given color and finally the computation of the modified Hausdorff Distance between set X (reference) and Y (test)

irises in the databases at hand) while the Hue values for blue irises varies around $200^{\circ}$. Figure 5 shows one Hue distribution of each class.

In order to classify the two kinds of irises (brown and blue irises) we have used a simple Gaussian Mixture Model [12] with 3 Gaussians for each class. Some images may contain eyelids occlusion; as eyelids fall in range of 300 to $360^{\circ}$, these images can affect the Hue distribution by introducing a new peak (see Figure 5 right). As the discrimination between the 2 classes is mainly due to other values range of the distribution, eyelids don't affect the classification capacity of the GMM.

In order to pre-classify an iris test image, we select the class (brown or blue) corresponding to the GMM giving the highest probability. After this preclassification, only comparisons between reference and test irises belonging to the same class are performed (the reference image is stored with its associated class label). In case no comparison is performed, a maximum score is given to the corresponding test image.
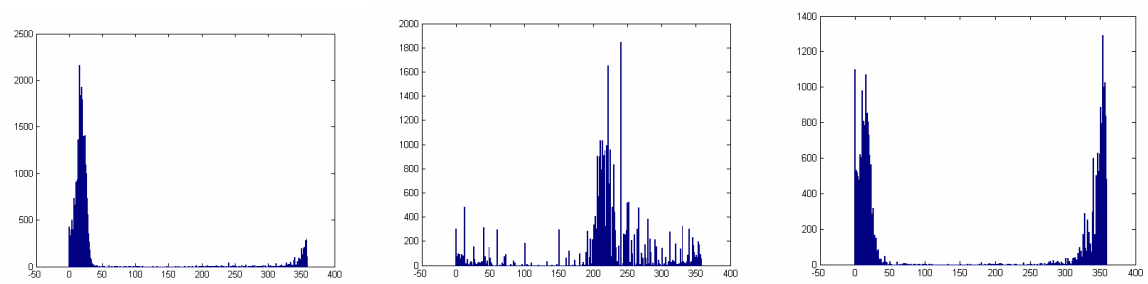

Fig. 5. Brown iris Hue histogram (left image), blue iris Hue histogram (middle image) and brown iris with strong Eyelids presence (right image) 


\section{Evaluation}

\subsection{Normal Light Iris Databases}

There are few iris databases acquired under normal light condition. In this work we have used a quite small database acquired by our team, called IRIS_INT as development set and we made the tests on UBIRIS database.

IRIS_INT contains 700 images from 70 persons. There are between 7 and 23 iris images per person. The images have been taken with a flash at a distance of 20-30 cms; the camera has a $23 \mathrm{~mm}$ focus, the image resolution is $2048 \times 1536$ and the captured irises have 120-150 pixels of radius. Some variations in lighting and position have been introduced in the database. We have chosen to capture only the left iris of each person. In fact, we acquired the top left quarter of the subject's face. This database includes some other variations including out of focus images, eyelids and eyelashes occlusions, lenses and eyeglasses effects.

UBIRIS iris database was developed by the Universidade da Beira Interior, with the aim of testing the robustness of various iris recognition algorithms to degradations. Several intra-class variations or image degradations, such as illumination, contrast, reflections, defocus, and occlusions are present in this database. The database contains 1,877 gray-level images from 241 persons, captured in two different sessions. Some images are shown in Figure 6.

In the first session, noise factors are minimized as the irises are acquired indoor, in a controlled illumination environment (dark room). Images are available at two different resolutions, a high resolution $(800 * 600)$ and a low resolution $(200 * 150)$. In our experiments we used the smallest resolution available in order to simulate PDA or smartphone limitation in terms of resolution. Indeed, if we consider the half top of a face acquired with a PDA or smartphone camera of 2 megapixels, an iris in average will be represented by an image of size $400 * 300$ which doubles the resolution that we consider.

We have used 1727 images from the 1,877 which are available. The deleted images correspond to very degraded images (closed eye, blurring, occlusion).
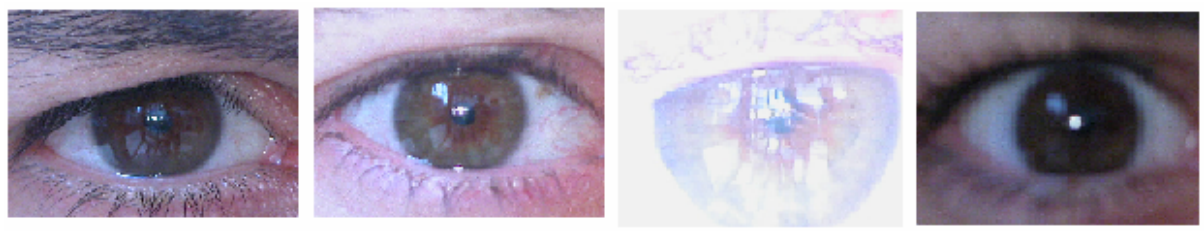

Fig. 6. Some low quality images (highly covered by eyelids and spot lights) acquired in session 2 (uncontrolled images) of the UBIRIS database

\subsection{Experiments}

We have used two reference systems in order to benchmark our system although those systems were developed and optimized on a different data (irises acquired under near infrared illumination). These two systems; Masek [6] from the University of Western 
Australia, and OSIRIS developed under the BioSecure European Network of Excellence [11] correspond to implementations of Daugman's work [7] although none of them can pretend to be equivalent to Daugman's system in terms of optimization and recognition performance.

In the present work, all developments are made on IRIS_INT database while tests are made on UBIRIS database. We developed two different protocols (or scenarios) on UBIRIS. In the first protocol, called "controlled scenario", we only consider images from the first session, that is images acquired indoor, in a dark room under controlled illumination conditions. In the second protocol, "called uncontrolled scenario", images of the first session are used as references while those of the second session are used as test images. In both cases, only one image is considered as reference.

Development experiments. We made all possible comparisons between images of IRIS_INT database. It has allowed us to optimize some parameters in the system such as the number of colors in the compressed images. We considered from 30 to 300 colors in the color map, and the best results were obtained for color maps of 60 colors. Indeed, if too many colors are considered, the similarity between reference and test images of the same person is lost. On the other hand if too few colors are considered the essential information to discriminate a client and an impostor will be also lost. Also in this case too many pixel positions will be associated to each color in the color map, which increasing considerably the computational load when computing the modified Hausdorff distances.

Controlled scenario. For the controlled scenario on the UBIRIS database we obtain $1.2 \%$ of EER, and $2.1 \%$ of FRR with $0.1 \%$ of FAR. These results are very encouraging as both OSIRIS and MASEK perform poorly on the same data (of course on grey-level images), from 5 to 10 times worst than our color-based approach (see Table1). Such a difference between systems can only be explained by the fact that color carries some information which is definitively lost when we just use grey-level images and texture information only. Indeed, the quality of texture information is bad and not sufficient for such analysis. Also, the bad performance of both Masek and OSIRIS confirm that texture-based methods suffer greatly from the low resolution of the images (small size of irises, a radius lower than 50 pixels).

Uncontrolled scenario. Uncontrolled illumination changes affect both color and texture of the image. Tests show a huge performance degradation compared to the controlled scenario, up to $22 \%$ of FRR at $0.1 \%$ of FAR for our color-based approach that still outperforms significantly OSIRIS and Masek. Table 1 summarizes the performance obtained in each scenario and with the three different systems.

Table 1. Benchmarking performances on UBIRIS database under different scenarios with Color-based system, OSIRIS and Masek systems

\begin{tabular}{|c|c|c|c|c|}
\hline & \multicolumn{2}{|c|}{ Controlled scenario } & \multicolumn{2}{|c|}{ Uncontrolled scenario } \\
\hline & EER & FRR & EER & FRR \\
\hline Color & 1.2 & 2.1 & 8 & 22 \\
\hline OSIRIS & 5 & 10 & 14.5 & 40 \\
\hline Masek & 7.1 & 20 & 25 & 80 \\
\hline
\end{tabular}


To cope with bad quality images of this uncontrolled scenario, like images which are covered by a high number of spot lights as shown in Figure 6, we introduce a quality measure on iris images at the acquisition step. This way images would be rejected right after acquisition and thus not processed by the system; system performance would this way be increased. In fact, in the UBIRIS database brown irises suffer much more from illumination changes than blue irises: for brown irises the 'red' colors (range $0^{\circ}-50^{\circ}$ ) turn to purple in most cases (less than $360^{\circ}$ ). Therefore, to detect bad quality images we only use the GMM trained on brown irises (acquired in the controlled scenario) for the two classes separation by applying a threshold on the probability given by the GMM.

Figure 7 shows the distribution of the resulting GMM probabilities on brown irises, when considering all the brown iris images from both the first session (red points) and the second session (blue points). The principle to select test images by the proposed quality measure is the following: only irises that have a probability comparable to those obtained on iris images of the first session (controlled scenario) are kept. Irises from the first session have a minimum probability of 0.42 ; so we fixed the selection threshold at 0.4 . This means that irises with a probability lower than 0.4 are rejected and not considered in the test process. Using this selection process, $80 \%$ of brown irises from the second session (uncontrolled scenario) are rejected. The EER falls from $8 \%$ to $1.5 \%$, and FRR reaches $3.8 \%$ at FAR of $0.1 \%$ (versus $22 \%$ without considering the selection process).

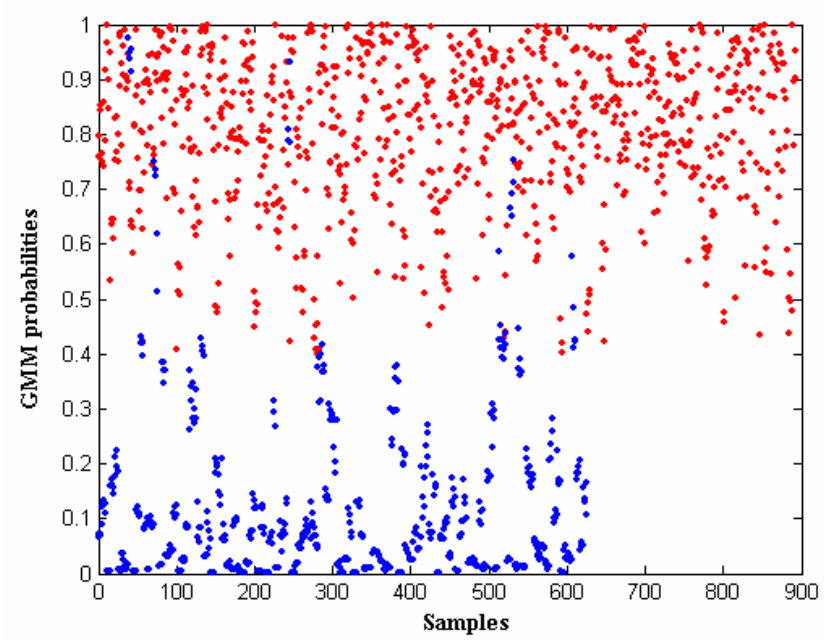

Fig. 7. GMM probabilities on brown irises from session1 (Red) and Session2 (Blue)

\section{Conclusions}

In this paper we presented a novel iris verification method entirely based on the color information, using the minimum variance principle for iris color reduction and a modified Hausdorff distance for matching. We also proposed a pre-selection method 
based on the use of Gaussian Mixture Models that characterize two classes of colors (blue and brown or more generally clear and dark) for iris images encoded in the Hue axis of the HSV color space. These models are also used to detect images corrupted by a variety of strong illumination effects. Our tests show that in the controlled acquisition mode our system outperforms significantly two iris reference systems based on texture information. Nevertheless, in uncontrolled conditions, tests show a huge decrease in performance for our method although it remains better than those obtained with texture-based systems (22\% vs. $40 \%$ and $80 \%)$. In order to cope with image degradation, typical in the uncontrolled scenario, we proposed a quality measure based on the GMMs above mentioned to reject bad quality images. This selection process drastically improves the results of our approach reducing the error rates roughly by a factor 6 , while rejecting $80 \%$ of the images acquired in uncontrolled conditions.

Future work will be focused on fusion strategies of texture-based systems and our color-based approach on images acquired under normal illumination conditions.

\section{References}

1. http://www.iridiantech.com/index2.php

2. Emine Krichen, M., Mellakh, A., Garcia-Salicetti, S., Dorizzi, B.: Iris Identification Using Wavelet Packets. In: 17th International Conference on Pattern Recognition (ICPR 2004), Cambridge, UK, 23-26 August 2004, vol. 4, pp. 335-338 (2004)

3. Sun, Z., Wang, Y., Tan, T., Cui, J.: Improving iris recognition accuracy via cascaded classifiers. IEEE Transactions on Systems, Man, and Cybernetics, Part C 35(3), 435-441 (2005)

4. http://iris.di.ubi.pt

5. Wildes, R.: Automated iris recognition: An emerging biometric technology. Proceedings of the IEEE 85 (9), 1348-1363 (1997) Awarded IEEE Donald G. Fink Prize Paper Award

6. http://www.csse.uwa.edu.au/ pk/studentprojects/libor/

7. Daugman, J.: How iris recognition works. IEEE Transactions on Circuits and Systems fo Video Technology 14(1) (January 2004)

8. Dubuisson, M.-P., Jain, A.K.: A modified Hausdorff distance for object matching. Pattern Recognition (1994). In: Conference A: Computer Vision \& Image Processing, Proceedings of the 12th IAPR International Conference, 9-13 October 1994, vol. 1, pp. 566-568 (1994)

9. Mathworks, Inc., Matlab Image Processing Toolbox, Ver. 2.2 Natick, MA (1999)

10. Smith, A.R.: Color Gamut Transform Pair. Computer Graphics 12(3), 12-19 (1978)

11. http://www.biosecure.info 